\title{
Processo de Desenvolvimento para Jogos Eletrônicos Educacionais: uma Revisão de Literatura
}

\author{
Development Process for Educational Computer Games: a Literature Review
}

\author{
João Coelho Neto \\ Colegiado de Matemática \\ Centro de Ciências Humanas e da Edu- \\ cação \\ Universidade Estadual do Norte do \\ Paraná - Campus de Cornélio Procópio \\ joaocoelho@uenp.edu.br
}

\author{
Sheila Reinehr \\ Escola Politécnica \\ Programa de Pós-Graduação em \\ Informática - PPGIa \\ Pontifícia Universidade Católica do \\ Paraná \\ sheila.reinehr@pucpr.br
}

\author{
Andreia Malucelli \\ Escola Politécnica \\ Programa de Pós-Graduação em \\ Informática - PPGIa \\ Pontifícia Universidade Católica do \\ Paraná \\ malu@ppgia.pucpr.br
}

Resumo A área de jogos eletrônicos educacionais tem crescido rapidamente. Entre os fatores responsáveis por esse crescimento evidenciam-se a diversidade e a possibilidade de integração que estes proporcionam entre o campo tecnológico e os métodos educacionais. Todavia, entende-se que o desenvolvimento desses jogos eletrônicos é uma tarefa complexa, uma vez que requer a colaboração efetiva de uma equipe multidisciplinar que precisa dominar habilidades técnicas e educacionais. Com base nessas atribuições, a proposta deste artigo é investigar, por meio de uma revisão sistemática de literatura, quais os processos de desenvolvimento de software que estão sendo utilizados nos jogos eletrônicos educacionais e se há algum modelo de processo que direcione a equipe à compreensão das habilidades cognitivas e psicomotoras. A revisão abordou trabalhos publicados no periodo 1998-2014, em sete bases de dados científicas diferentes. Foram encontrados 956 artigos. Após aplicar o critério de exclusão, o conjunto foi reduzido para 55 artigos, os quais foram lidos integralmente, revisados e analisados e apenas 4 artigos correspondiam claramente aos critérios definidos para a realização dessa revisão sistemática. Os trabalhos foram classificados de acordo com o tipo de modelo de desenvolvimento de software utilizado. A análise possibilitou constatar que há uma carência de pesquisas voltadas aos processos de desenvolvimento de jogos eletrônicos educacionais. Também foi possivel identificar que há processos de desenvolvimento para jogos eletrônicos educacionais adaptados de processos de desenvolvimento já existentes, tais como: cascata, espiral e RUP. Não foram encontrados trabalhos nesta pesquisa que contemplem a questão de processos que direcionam a equipe à compreensão das habilidades cognitivas e psicomotoras .

Palavras-Chave: Jogos Eletrônicos; Revisão Sistemática de Literatura; Jogos Eletrônicos Educacionais; Processo de Desenvolvimento de Software; Habilidades Cognitivas; Habilidades Psicomotoras.

\footnotetext{
Abstract The area of educational electronic games has been growing quickly. Among other factors responsible for such growth we may evidence the diversity and the possibility of integration that these games allow between the technologic field and the educational methods. However, we understand that the development of these electronic games is a complex task, once it requires the effective collaboration of a multidisciplinary team that must dominate the technical and educational abilities. Based on these attributions, the purpose of this paper is to investigate, through a systematic review of the literature, which are the software development process that are being used in the educational electronic games, and whether or not there a model of the process that directs the team toward the comprehension of the cognitive and psychomotor abilities. The review approached the works published within the period of 1998-2014, on seven different scientific data bases. We have found 956 papers. Upon applying the criteria of exclusion, the block of papers was reduced to 55, which were fully read, reviewed and analyzed, and only 4 papers corresponded clearly to the criteria established for the execution of such
} 
systematic review. The works were classified as per the type of a model for the development of software used. The analysis allowed us to find that there is a lack of researches focused on the developmental process of educational electronic games. It was also possible to identify that there are developmental process for adapted educational electronic games of already existing development, such as: cascade, spiral and RUP. We have not found works in this research that contemplate the matter of processes that guide the team to the comprehension of the cognitive and psychomotor abilities.

Keywords: Electronic Games; Systematic Review of Literature; Educational Electronic Games; Software Development Process; Cognitive Abilities; Psychomotor Abilities. 


\section{Introdução}

Nos últimos 40 anos, os jogos eletrônicos em âmbito mundial vêm substituindo os jogos mais tradicionais, as atividades de lazer e outras atividades de entretenimento. Como consequência, há uma transformação no modo como as pessoas utilizam seu tempo livre [1].

Os jogos possibilitam uma nova abordagem do mundo do entretenimento, da informação e da educação, visto que estes instrumentos podem possibilitar a diversão, gerando um novo contexto de comunicação, disputando a atenção de crianças, jovens e adultos com outros espaços de lazer, de organização e apropriação do conhecimento, como a escola [2].

Além disso, os jogos eletrônicos tornaram-se uma estratégia que possibilita a aprendizagem, podendo ser utilizada em diferentes contextos educacionais [3]. Isso se dá porque computadores, videogames e jogos eletrônicos educacionais detêm facilmente a atenção dos estudantes. No entanto, o uso destes recursos em sala de aula não é fácil, visto sua diversidade de utilizações. Por isso, há uma necessidade de se definir estratégias de ensino pertinentes aos recursos tecnológicos utilizados [4].

Por esses motivos, os jogos eletrônicos educacionais têm sido aplicados em uma diversidade de áreas de pesquisa, visto a multivariedade de contribuições neles potencializadas [5], instrumentos estes fortemente recomendados na área da informática na educação.

Esta multivariedade possibilita a união com as diversas áreas do conhecimento, englobando desde partes do próprio desenvolvimento (game design, programação, arte, músicas, efeitos sonoros); periféricos de entrada e saída; área da informática (inteligência artificial (IA), redes e engenharia de software); e as áreas da educação.

Com isso, a área de jogos eletrônicos educacionais tem crescido rapidamente e devido essa diversidade há a necessidade de integração entre a tecnologia e os métodos educacionais [6;7].

Devido à necessidade desta integração, a construção de jogos eletrônicos educacionais não é uma tarefa fácil. Esta tarefa requer a colaboração efetiva de equipes pedagógicas que saibam como projetar a aprendizagem de conteúdos, estratégias, designers de jogos e outros profissionais da área de tecnologia, que dominem as habilidades técnicas e conhecimentos necessários para a construção de jogos eletrônicos sofisticados, além de uma equipe que auxilie na avaliação desses produtos em âmbito computacional e educacional [8].

A partir dessas colaborações, as ações educacionais devem ser utilizadas no desenvolvimento de jogos eletrônicos, pois essas ações são capazes de minimizar a carência de recursos educacionais e cognitivos no desenvolvimento do jogo eletrônico final, especialmente, os educacionais.
Destarte, no desenvolvimento desses jogos eletrônicos educacionais, uma das dificuldades encontradas no alcance de sua qualidade parece estar ligada ao fato de que no processo de desenvolvimento, percebe-se uma diferença significativa entre as intenções que designers, programadores e professores manifestam sobretudo no que compete às funções pedagógicas desses jogos $[9 ; 10]$.

Motivados por estas diferenças, foi realizada uma pesquisa inicial buscando informações sobre o desenvolvimento de jogos voltados à educação. Um estudo sistemático realizado [11] apontou que, em um evento específico na área de Informática na Educação, 5,4\% dos artigos, ou seja, 48 de 885 apresentavam trabalhos sobre jogos, porém, apenas um artigo [12] contemplava um processo para o desenvolvimento de jogos eletrônicos educacionais, porém, percebeu-se que não considerava questões educacionais (cognitivas) em suas etapas.

Evidenciou-se assim, a necessidade de estudo nesta temática, visto que, o uso dos jogos eletrônicos voltados ao processo educacional está crescendo, podendo ter resultados benéficos para o processo de ensino e aprendizagem, e contribuir para a área da informática aplicada à educação, como componente didático-metodológico em sala de aula. Conforme Vosgerau [13] "[...] a tecnologia educacional é um campo de estudo cuja preocupação principal é a melhoria do ambiente educacional com vistas a facilitar o processo de ensino-aprendizagem, da mesma forma que se propõe a criar métodos e técnicas para possibilitar o desenvolvimento e a produção de ambientes de aprendizagem, sejam eles tecnológicos ou não" (p.273).

Portanto, os recursos tecnológicos de aprendizagem são fundamentais para uma educação de boa qualidade, visto que estas propostas estão presentes em vários setores da educação e sua utilização tem evoluído ao longo dos tempos, especialmente nos modos convencionais e didáticos de ensino [14]. Entretanto, para que isso seja possível, é necessário que haja uma preocupação durante o desenvolvimento dos jogos com as questões educacionais, principalmente as cognitivas.

Portanto, ao explorar as dimensões pedagógicas, cognitivas, psicomotoras e motivacionais, espera-se que os jogos eletrônicos educacionais possam ser utilizados como elementos auxiliares nos processos de ensino e de aprendizagem.

Diante deste contexto, a proposta deste artigo é analisar, por meio de uma Revisão Sistemática de Literatura (RSL), quais são os processos de desenvolvimento de software que estão sendo utilizados no desenvolvimento de jogos eletrônicos educacionais e se esses processos incluem em suas etapas aspectos educacionais ou cognitivos.

Pretende-se assim, responder as seguintes questões de pesquisa: 1) Quais processos de desenvolvimento de software estão sendo utilizados para a construção de jogos eletrônicos educacionais?; 2) Há algum processo de 
desenvolvimento que direcione a equipe, em suas atividades, à compreensão das habilidades cognitivas e psicomotoras?

Para uma melhor compreensão desta temática, este artigo está organizado da seguinte maneira: a seção 2 apresenta o aporte teórico referente aos processos de desenvolvimento de softwares e de jogos eletrônicos, além da observação da contribuição desses jogos no desenvolvimento cognitivo do aprendiz; na seção 3, a abordagem metodológica é descrita; na seção 4, os resultados são apresentados; na seção 5 são discutidos os resultados e a seção 6, são apresentadas as conclusões, limitações e trabalhos futuros deste artigo.

\section{Aporte Teórico}

Esta Seção apresenta alguns conceitos para o melhor entendimento deste trabalho, envolvendo: processos de desenvolvimento de software, jogos eletrônicos educacionais, habilidades cognitivas e psicomotoras, além de discutir sobre o uso de jogos eletrônicos na educação.

\subsection{Processo e/ou Modelo de Desenvolvimen- to de Software}

De acordo com Humphrey [15], um processo de desenvolvimento de software é um conjunto de atividades da engenharia de software recomendadas para transformar requisitos de um usuário em um software.

Assim, um processo de desenvolvimento de software compreende várias atividades que têm como objetivo final a produção de um produto de software [16]. O desenvolvimento de software pode ser extremamente complexo e há várias maneiras de se executar as diversas atividades envolvidas. Portanto, a definição de um processo de desenvolvimento de software pode auxiliar os profissionais a executarem estas atividades de maneira ordenada, facilitando, assim, o entendimento das ações de desenvolvimento por parte da equipe [15].

Os modelos de ciclo de vida do software descrevem basicamente as principais etapas do desenvolvimento de um software, abrangendo o levantamento de requisitos até sua manutenção. Há vários modelos de ciclo de vida, mesmo porque, esses ciclos foram se adaptando conforme as necessidades específicas de desenvolvimento de software no decorrer dos anos.

O Modelo Cascata ou Waterfall Model, também chamado de Ciclo de Vida Clássico ou Ciclo de Vida do Software, é o mais antigo dos modelos. Esse modelo foi desenvolvido nos anos 70 do século XX por Winston Royce, constituído pelas atividades de Definição de Requisitos, Projeto, Implementação, Integração e Operação [16].

O Modelo Incremental ou Iterativo é semelhante ao Modelo Cascata. Os requisitos, conceitos de software e sistema são inicialmente identificados e, posteriormente, seguem as demais etapas de desenvolvimento de softwa$r e$, porém aplica sequências lineares de uma forma racional à medida que o tempo passa. Este modelo foi inicialmente proposto por Boehm, que ao invés de apresentar uma sequência de atividades com retorno entre elas, todo o processo é representado em forma de espiral, sendo formado por loops. Cada loop representa uma fase do processo de software e está dividido em quatro setores: definição dos objetivos; avaliação e redução de riscos; desenvolvimento e validação; e planejamento.

Há também o Rational Unified Process (RUP), que é um processo derivado da Unified Modeling Language (UML) e do Processo Unificado (PU) de Desenvolvimento de Software Associado (DSA). O RUP é um processo de engenharia desenvolvido e comercializado pela Rational Software [17].

Esse processo é uma abordagem disciplinada para a atribuição e gestão de tarefas e responsabilidades em uma organização de desenvolvimento e é capaz de capturar as boas práticas no desenvolvimento de software moderno, apresentando uma estrutura adequada para uma ampla gama de projetos e organizações [16].

Atualmente muitas organizações de desenvolvimento de software também estão utilizando Metodologias Ágeis, que têm apresentado ótimos resultados perante pesquisadores e usuários. As Metodologias Ágeis incluem: Extreme Programming (XP), Feature DrivenDevelopment (FDD) - Desenvolvimento Guiado por Características, Crystal, Scrum e Desenvolvimento de Sistemas Dinâmicos [18]. Constituem um grupo de metodologias entre si, porém, caracterizados por princípios comuns, baseando-se em trabalhos cooperativos e na documentação escrita. Estes princípios foram conhecidos por um documento chamado de Manifesto Ágil ${ }^{1}$, no qual encontram-se doze princípios de um software ágil.

Conforme identificado, os processos de desenvolvimento de software são de suma importância para o desenvolvimento de qualquer tipo de software, inclusive, os jogos eletrônicos.

Compreendendo a importância de um processo de desenvolvimento, na próxima subseção serão abordadas, as contextualizações relacionadas ao processo de desenvolvimento de um jogo eletrônico educacional e o impacto de sua abordagem no processo de ensino e de aprendizagem.

\subsection{Desenvolvimento de Jogos Eletrônicos Educacionais}

O desenvolvimento de um jogo eletrônico é realizado por equipes multidisciplinares que são desafiadas a criar

\footnotetext{
${ }^{1}$ Disponibilizado em http://agilemanifesto.org - Versão Inglês

Disponibilizado em http://agilemanifesto.org/iso/ptbr/ - Versão Português
} 
um produto de entretenimento que possibilite diversão e imersão em um ambiente interativo, para um determinado público alvo [19].

Ainda em Souza e Kafure [19] abordam que dada a complexidade que envolve o trabalho da equipe em desenvolver jogos eletrônicos, o cumprimento de tarefas e o comprometimento do grupo são fundamentais para um bom produto final. Por isso, empresas de desenvolvimento de jogos, conforme adquirem experiência, passam a modelar um processo próprio para a criação de jogos, que com o tempo, vai sendo aperfeiçoado para atender as necessidades encontradas.

Consequentemente, o acesso a uma metodologia de desenvolvimento é restrita ou não trivial, e os desenvolvedores de jogos costumam, por exemplo, "não incluir em seu escopo, o planejamento do jogo e partir direto para sua implementação, o que tem como consequência altos níveis de retrabalho" (p.125) [20].

Em relação aos jogos eletrônicos educacionais, além do retrabalho devido à falta de definição e utilização de um processo, existe ainda a falta de conhecimento em relação às questões educacionais que deveriam ser consideradas durante o seu desenvolvimento.

Além disso, Gomes e Wanderley [9] apontam que há poucos sistemas voltados à educação que utilizam modelos educacionais e construtivistas para modelar ações dos usuários com o software a ser desenvolvido.

Dessa forma, ainda em Gomes e Wanderley [9] uma questão importante, presente no desenvolvimento de produtos educacionais, compreende as especificações educacionais feitas nesses modelos, pois, muitas vezes, estas especificações parecem pouco integralizadas nesse desenvolvimento.

Entretanto, se as equipes de desenvolvimento não identificarem ou conhecerem essas especificações educacionais ou ações cognitivas, dificilmente irão projetar jogos que possam realmente produzir recursos para auxiliar o processo de ensino e de aprendizagem.

\subsection{Habilidades Cognitivas e Psicomotoras}

$\mathrm{Na}$ área de jogos eletrônicos educacionais, o uso das habilidades cognitivas e psicomotoras pode fazer a diferença em um jogo, visto que, estes podem ser utilizados com muito sucesso, já que, auxiliam nas habilidades cognitivas dos envolvidos no processo de cognição [21].

A cognição é o ato ou o processo de conhecer, que visa reconhecer as seguintes habilidades: percepção, atenção e consciência, memória, representação do conhecimento, linguagem, resolução de problemas e criatividade, e tomada de decisão e raciocínio [22]. Assim, a cognição é um importante elemento quanto as necessidades de aprendizagem dos indivíduos, e com isso, as habilidades cognitivas adquiridas são fatores importantes no contexto escolar e na formação de cidadãos mais reflexivos e críti$\cos [23]$.
Dessa forma, as habilidades cognitivas descritas como aporte teórico neste trabalho, tiveram como base Sternberg [22], e são descritas como:

1. Percepção: é o conjunto de processos pelos quais é possível reconhecer, observar e entender as sensações provenientes dos estímulos.

2. Atenção e Consciência: é o meio pelo qual se processa ativamente uma quantidade limitada de informação, a partir da enorme quantidade de informação disponível por meio dos sentidos, da memória armazenada e de outros processos cognitivos.

3. Memória: é o meio pelo qual retemos e nos valemos de nossas experiências passadas para usar essas informações no presente.

4. Linguagem: é o uso de um meio organizado de combinação de palavras a fim de criar comunicação.

5. Resolução de Problemas e Criatividade: a resolução de problemas são esforços para superar obstáculos que obstruem o caminho para uma solução, e a criatividade é um processo de se produzir algo que seja original e válido ao mesmo tempo.

7. Tomada de Decisões e Raciocínio: julgamento e tomada de decisões são usados para selecionar entre diversas escolhas ou para avaliar oportunidades.

8. Raciocínio: é o processo de chegar a conclusões com base em princípios e provas.

$\mathrm{Na}$ área de jogos eletrônicos educacionais, estas habilidades podem ser de extrema importância, em razão destas características os tornarem extremamente atrativas para serem utilizadas como instrumento didáticopedagógico.

Além disso, para que os jogos eletrônicos educacionais possam configurar-se numa forma lúdica de desenvolver as habilidades cognitivas, os conteúdos devem ser explorados de uma forma atrativa e motivadora, para que essas informações possam potencializar a criatividade e iniciativa na busca de estratégias e mecanismos eficientes para resolver as situações-problemas suscitadas [24].

Estudos mostram que a utilização dos jogos eletrônicos educacionais que preocupam-se com as habilidades cognitivas e motoras em seu contexto podem auxiliam no desenvolvimento destas habilidades com alunos que possuam alguma deficiência ou não [25].

Por isso, além das habilidades cognitivas, outras habilidades podem ser fundamentais para o contexto educacional, como as psicomotoras. Essas habilidades são descritas, de acordo com Meur e Staes [26], como: coordenação motora, lateralidade, orientação espacial e orientação temporal, sendo apresentadas a seguir:

1. Esquema corporal: é a representação relativamente global, científica e diferenciada que a criança tem em seu próprio corpo, sendo um elemento básico indispensável para a formação da personalidade [26;27].

2. Lateralidade: naturalmente se define uma dominância lateral: será mais forte, mais ágil do lado direito ou do lado esquerdo. A lateralidade corresponde a dados 
neurológicos, mas também é influenciada por certos hábitos sociais [26].

3. Orientação Espacial: é a tomada de consciência da situação do seu próprio corpo; a tomada de consciência da situação das coisas entre si; a possibilidade de organizar-se perante o mundo que o cerca, de organizar as coisas entre si, de colocá-las em um lugar, de movimentá-las (p. 13) [26].

4. Orientação Temporal: é a capacidade de se situar em função das sucessões dos acontecimentos e da duração de intervalos. Esta habilidade visa apropriar as seguintes ações: tempo, ritmo regular e irregular (aceleração, freada); cadência rápida, decadência lenta (diferença entre a corrida e o andar), além de propiciar a função da duração dos intervalos.

Assim, a psicomotricidade propõe colocar o indivíduo em uma situação que lhe permita viver emocionalmente o espaço, os objetos e a relação com os outros. Essa relação é permitida por meio dos jogos sensório motores que permitem a exploração das sensações do corpo e do jogo simbólico, que é o aspecto das atividades de representação, considerando o desenvolvimento integral do indivíduo, ou seja, os aspectos: motores, afetivos e cognitivos, prevenindo dessa forma as dificuldades de aprendizagem [28].

Desse modo, um jogo eletrônico educacional, desenvolvido com base nessas habilidades (cognitivas e psicomotoras) também pode estimular o desenvolvimento cognitivo e o desenvolvimento de aptidões e habilidades motoras [29]. Além disso, estas habilidades são processos cognitivos que envolvem a reflexão.

Essas reflexões podem trazer resultados benéficos no processo de ensino e aprendizagem visto suas potencialidades no âmbito escolar, possibilitando assim, novo processo de aprendizagem que antes não seria possível sem o uso dos jogos eletrônicos educacionais $[20 ; 21 ; 23 ; 24 ; 25$; 28; 29;30].

Estes processos possibilitam o ato de pensar e analisar uma decisão por suas caracterizações em sua contextualização e tem um elevado valor para a equipe de desenvolvimento, porque podem influenciar diretamente as ações explícitas no produto final [31].

Identificando essas percepções referentes à importância da integração das habilidades cognitivas e psicomotoras em um jogo eletrônico educacional, a próxima subseção aborda a relação benéfica do uso dos jogos eletrônicos para o processo de ensino e de aprendizagem.

\subsection{Jogos eletrônicos na Educação}

Os jogos eletrônicos educacionais são atividades agradáveis com objetivos educacionais especializados para desenvolver o pensamento e a aprendizagem, e assim tornam-se um poderoso recurso para a construção do conhecimento [12;32].
Além disso, proporcionam situações lúdicas e possuem objetivos pedagógicos especializados para o desenvolvimento do raciocínio e do aprendizado, fatores principais para o uso desses instrumentos no âmbito educacional [33]. Novak [34] aborda que os jogos eletrônicos educacionais são aqueles criados para ensinar enquanto distraem.

Corti [35] aborda que os jogos eletrônicos criam um ambiente no qual os usuários aprendem a explicar, descrever, construir, comparar, analisar, acessar e avaliar.

Dessa maneira, esses jogos compartilham experiências que envolvem seus participantes em um processo cognitivo e emocional, proporcionado pelo uso de histórias narrativas, drama, humor e desenvolvimento de personagens.

Além disso, os jogos proporcionam a melhora da flexibilidade cognitiva, pois funcionam como uma ginástica mental, aumentando a rede de conexões neurais e alterando o fluxo sanguíneo no cérebro quando em estado de concentração [30].

A partir dessas contextualizações, identifica-se que a utilização de jogos eletrônicos na educação pode proporcionar ao aluno motivação, desenvolvendo também hábitos de persistência no enfrentamento de desafios e realização de tarefas, possibilitando a integração das habilidades cognitivas em um contexto eletrônico de aprendizagem.

\section{Método}

O método utilizado nesta pesquisa é a Revisão Sistemática de Literatura (RSL), que é um meio de identificar, avaliar e interpretar trabalhos disponíveis relevantes para uma questão de pesquisa específica, área temática ou algum fenômeno de interesse [36]. Na RSL algumas etapas devem ser seguidas para que seu objetivo seja contemplado e reproduzido. Wholin et al. [37], apresentam as seguintes etapas: 1. Identificação da necessidade da revisão; 2. Especificação da questão de pesquisa; 3. Desenvolvimento do protocolo de revisão. Kitchenham [33] aponta três fases principais para uma revisão: planejamento da revisão, condução da revisão e elaboração de relatórios, dessas fases principais, estágios associados são definidos para uma melhor estruturação no planejamento e condução da revisão.

Para esta RSL, foram seguidas a combinação das etapas propostas por Kitchenham [33] e por Wholin et al. [37], visto que, as etapas propostas por cada um, assemelhavam-se em uma estruturação de identificação, planejamento e condução para a análise dos dados de uma revisão sistemática, assim, estruturando na seguinte combinação de etapas e associações: planejamento da revisão, identificação e necessidade da pesquisa, seleção dos estudos primários e classificação dos resultados, conforme Figura 1. 


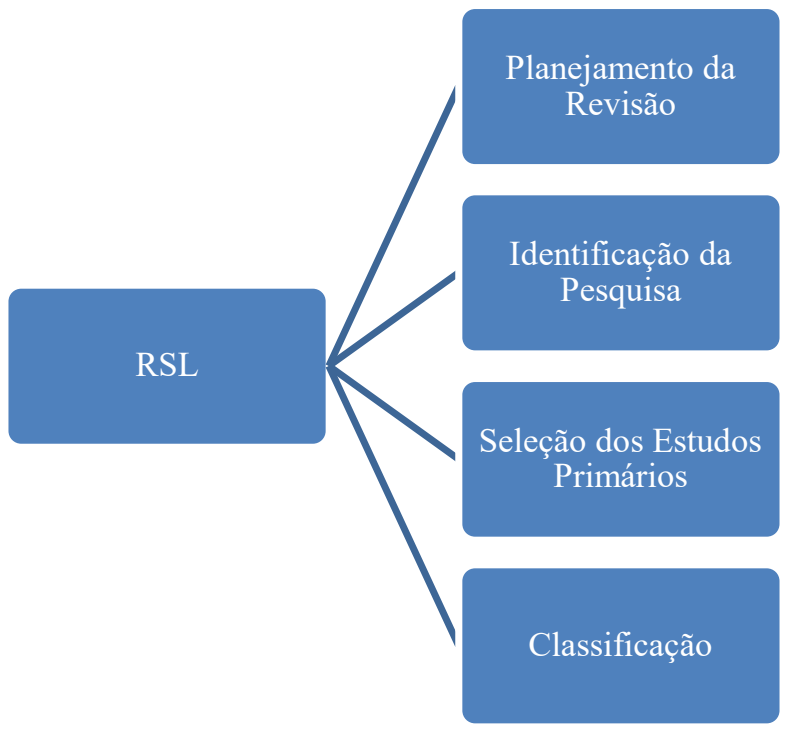

Figura 1 - Processo de Condução da Revisão

\subsection{Planejamento da Revisão}

O protocolo foi especificado com os procedimentos e métodos para a aplicação de uma RSL, com base em Kitchenham [33] e Wholin et al. [37]. Neste protocolo, o objetivo da revisão, questões de pesquisa, bases utilizadas para a revisão e critérios de inclusão e exclusão dos artigos são definidos.

Após a análise inicial, a primeira questão de investigação foi definida: 1) Quais processos de desenvolvimento de software estão sendo utilizados para a construção de jogos eletrônicos educacionais?

Com esta questão seria possível identificar como os jogos educacionais estão sendo desenvolvidos, porém não seria possível analisar se em cada processo identificado, há etapas dedicadas à compreensão de habilidades cognitivas e psicomotoras. Sendo assim, surgiu a segunda questão de investigação: 2) Há algum processo de desenvolvimento que direcione a equipe, em suas atividades, à compreensão das habilidades cognitivas e psicomotoras?

\subsection{Identificação da Pesquisa}

Este trabalho pesquisou artigos publicados em periódicos científicos, conferências, simpósios e workshops, durante o período de 1998 a 2014.

Fez-se uma pesquisa anterior ao período de 1998, porém, como é uma área relativamente nova comparada ao desenvolvimento de softwares tradicionais, identificou-se as primeiras pesquisas mais significantes na área do uso de jogos eletrônicos, principalmente na educação foi a partir de 1998, por isso, a escolha deste intervalo de busca.
A pesquisa foi realizada no período de janeiro a maio de 2014, porém, para a revisão final deste artigo, o intervalo foi estendido até dezembro de 2014, a fim de identificar se houveram novas publicações nesta área de pesquisa.

As bases de pesquisa utilizadas nesta revisão sistemática de literatura são apresentadas no Quadro 1, em ordem alfabética.

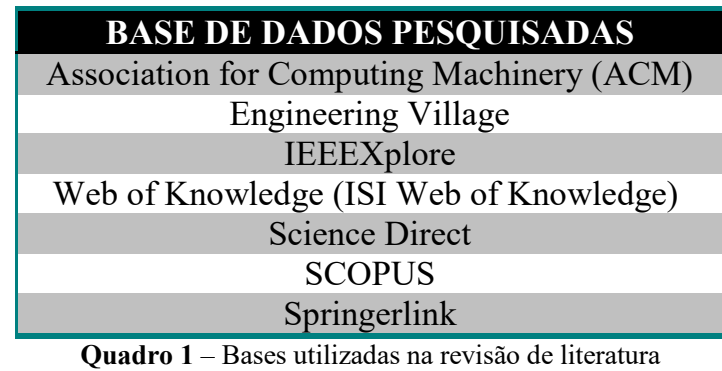

Identificadas as bases de buscas, as palavras-chave utilizadas na pesquisa foram: "educational games" and "development process". Utilizaram-se estas palavraschave, tendo como base, que esta combinação poderia abranger de forma ampla as pesquisas que estavam relacionadas com jogos eletrônicos educacionais e processos de desenvolvimento e também pelo fato de que, identificando processos de desenvolvimento de software e jogos educacionais, pode-se analisar quais são os processos de desenvolvimento para jogos eletrônicos educacionais que estão sendo utilizados e consequentemente, quais são as atividades que estão sendo contempladas nestes processos.

Desse modo, a busca foi realizada com ambas palavras-chave aparecendo simultaneamente, pois, visou identificar quais eram os processos de desenvolvimento utilizados nos jogos educacionais.

Feito a combinação destas palavras-chave, a pesquisa resultou em 956 artigos para a área de processos de desenvolvimento, referente a jogos eletrônicos educacionais, que formou a base para a seleção dos estudos primários.

\subsection{Seleção dos estudos primários}

A seleção dos estudos primários seguiu os critérios de inclusão e exclusão apresentados na Figura 2 e detalhados a seguir. 
Inclusão dos artigos que apontavam processos e desenvolvimento de jogos eletrônicos educacionais e eliminação de artigos com características que não apontam a temática pesquisada

\section{Eliminação dos Títulos Duplicados}

Leitura dos abstracts

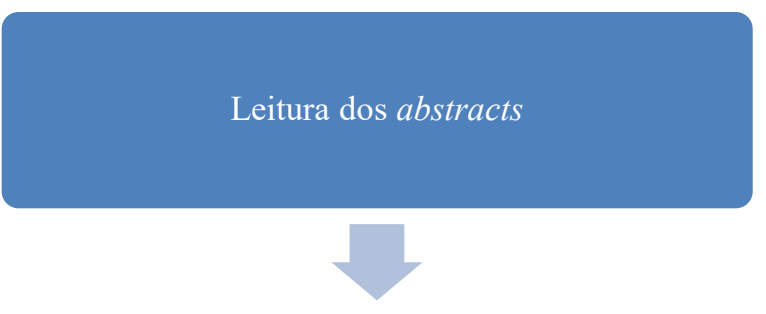

Exclusão dos artigos que não tratavam da temática

1. O primeiro passo após a identificação e inclusão dos artigos encontrados foi a eliminação dos artigos que tratavam das seguintes abordagens: artigos de revisão, design instrucional e artigos de um tipo específico de jogos, pois estes não fazem parte da análise desta revisão.

2. Eliminação dos títulos duplicados, pois o mesmo artigo apresentava em mais de uma base de dados.

3. Posteriormente, foi feita a leitura dos abstracts, no qual foram lidos e avaliados, usando o seguinte critério de exclusão:

3.1. Artigos que não abordavam claramente $o$ processo de desenvolvimento de jogos eletrônicos educacionais.

3.2. Artigos que apontavam processos de avaliação do uso de jogos eletrônicos em um contexto educacional, temática esta que não era o foco da pesquisa. $\mathrm{O}$ cerne principal era identificar se havia processos específicos para o desenvolvimento de jogos eletrônicos educacionais, e se nestes processos haviam etapas que proporcionassem às equipes ações educacionais para o entendimento e desenvolvimento de possibilidades educacionais no contexto do jogo final.

4. Após a leitura dos abstracts e exclusão dos títulos duplicados, foi feita a exclusão dos artigos que não trata- vam da temática pesquisada, o conjunto de artigos encontrados que apontavam a área pesquisada, dos 956 artigos encontrados foram reduzidos para 55 artigos, os quais foram lidos integralmente, revisados e analisados.

A leitura dos artigos na íntegra permitiu um novo processo de exclusão, pois a maioria dos artigos selecionados apresentava um processo de avaliação dos jogos eletrônicos educacionais, ou jogos prontos para aplicação em outras áreas do conhecimento. Com esta exclusão, o conjunto foi reduzido para 4 artigos que tratavam da abordagem pesquisada.

\subsection{Classificação}

Os artigos selecionados foram lidos novamente, buscando classificá-los de acordo com o tipo de processos definidos, na tentativa de extrair se esses artigos utilizavam processos existente na Engenharia de Software (ES) ou se o artigo apresentava um tipo de processo próprio, além de tentar identificar se nesses processos apontavam quais habilidades cognitivas e psicomotoras eram utilizadas nas etapas de desenvolvimento do jogo eletrônico educacional.

Dessa forma, ao fazer as leituras dos artigos, os resultados foram classificados de acordo com as questões de pesquisa: Quais processos de desenvolvimento de software estão sendo utilizados para a construção de jogos eletrônicos educacionais? Dessa forma, tentar identificar, por meio da primeira questão, a segunda pergunta dessa revisão: Há algum processo de desenvolvimento que direcione a equipe, em suas atividades, à compreensão das habilidades cognitivas e psicomotoras?

Para responder a primeira questão, elaborou-se um quadro (Quadro 2), com os tipos de processos encontrados nos artigos, os quatro artigos foram analisados verificando se os processos encontrados eram atividades próprias, criadas pelos autores ou atividades já existentes na Engenharia de Software adaptados ao contexto educacional, assim classificando-os com base na estrutura do quadro desenvolvido.

Para responder a segunda questão, como não foi encontrada na análise dos artigos etapas que contemplassem as habilidades cognitivas e psicomotoras, definiu-se um mapeamento de características em comuns nas etapas apresentadas pelos artigos analisados, a fim de identificar atividades em comum dos artigos analisados, apresentadas pelo Quadro 3.

Além de tentar responder a primeira e segunda questão desta revisão, algumas características sobre os artigos encontrados foram destacadas, a fim de mapear características de localização, pesquisadores e grupos de pesquisas encontradas nos artigos analisados.

Assim, na próxima seção as análises e resultados dos artigos encontrados são descritas e definidas. 


\section{Resultados}

Esta seção apresenta os resultados obtidos após a classificação dos artigos, o Quadro 2 apresenta os artigos classificados, respondendo a primeira questão dessa revisão.

\begin{tabular}{|cc|}
\hline MODELO & ARTIGOS \\
\hline Adaptação Cascata & Hodgson, Man e Lung [38] \\
\hline Adaptação Espiral & Moreno-Ger et al. [4] ${ }^{1}$ \\
\hline Adaptação RUP & Lee et al. [39] \\
\hline Próprio & Zaibon and Shiratuddin [40] \\
\hline
\end{tabular}

Quadro 2 - Classificação com base em sua adaptação

As próximas subseções apresentam uma descrição das etapas de cada um dos artigos, a fim de identificar de que forma podem contribuir para que as equipes de desenvolvimento de jogos eletrônicos identifiquem ou entendam ações educacionais que possam ser exteriorizadas nos jogos eletrônicos educacionais desenvolvidos.

\subsection{Modelos Encontrados}

Nesta seção, discutir-se-á os modelos encontrados, a fim de identificar possibilidades educacionais em seu contexto de desenvolvimento.

\subsubsection{Adaptado do modelo Cascata}

O trabalho de Hodgson, Man e Leung [38] propõe um modelo de sete fases que é uma combinação do modelo em cascata da engenharia de software tradicional e estruturas de gerenciamento de projetos, baseado no Project Management Body of Knowledge (PMBOK). A partir dessa combinação, a equipe desenvolveu o modelo incluindo atividades de design e produção multimídia e desenvolveu o "Seven-Stage Rapid Game Development Model". Estes estágios são classificados como:

- Stage 1: Understanding Content, um documento de trabalho é criado, o qual é passado para a equipe de criação para explorar as atividades do jogos eletrônicos educacionais.

- Stage 2: Forming a Learning Game Framework, baseado na discussão do estágio 1 , a equipe de criação propõe um brainstorm dos tipos de aprendizagem que o jogo deve proporcionar, focando no conteúdo a ser ensi-

\footnotetext{
${ }^{1} \mathrm{O}$ trabalho de Moreno-Ger et al. [4] apareceu em dois estilos de adaptação, visto que, esses autores apresentam um processo adaptado do estilo Espiral e RUP; porém, para análise, este foi classificado e analisado somente na subseção de estilo Espiral.
}

nado, discussão de jogos já construídos, e identificação de ações que possam ser utilizadas.

- No Stage 3: Bundling Learning Game Framework discute o estilo de entrega do jogo, incluindo história, natureza e tipos de atividades do jogo educativo a serem desenvolvidos.

- Stage 4: Consolidating Learning Game Framework, o responsável técnico e os líderes da equipe obtêm do professor do conteúdo abordado um conjunto de informações, e assim a documentação é gerada a partir do estágio 3. O diretor técnico negocia com o professor quando necessário.

- Stage 5: Formulating Production Plan, com base nos acordos gerados no estágio 4, o diretor técnico elabora um plano de produção que inclui divisão de trabalho e estrutura as tarefas para as equipes, o prazo para a produção modular, alocação de recursos e aquisição de equipamentos essenciais. Os membros de cada equipe recebem um conjunto de ações, com um cronograma para a conclusão.

- Stage 6: Executing Production Plan, neste estágio, as equipes de criação e de programação trabalham lado a lado. A equipe de conteúdo ajuda a revisar o conteúdo e pilotar os jogos educativos para avaliar se houve algum erro na programação. Membros da equipe interna de comunicação são essenciais para minimizar eventos imprevistos.

- Stage 7: Fine-tuning Learning Game, neste estágio, os jogos educativos são testados. Embora a escala dos testes dependa da quantidade antecipada de utilização, esperam-se várias pessoas participando desse estágio.

No geral, os estágios 1-5 objetivam a formulação de um plano para o desenvolvimento de um jogo educativo, enquanto, os estágios 6 e 7 desenvolvem o plano de produção.

\subsubsection{Adaptado do modelo Espiral}

No trabalho de Moreno-Ger, Martinez-Ortiz, Sierra and Fernández-Manjon [4], desenvolve-se o modelo de processo <e-Adventure>, formalmente conhecido como $<$ e-Game>. O recurso mais interessante desse modelo é a sua estrutura, visto que esta foi baseada nos modelos espiral ou iterativo e no Rational Unified Process (RUP), dividindo o processo em várias fases que podem ser repetidas tantas vezes quanto necessárias. Cada interação envolve as partes interessadas, tanto técnicas e não técnicas, que entregam produtos mais próximos do resultado final desejado.

Essa abordagem documental para o desenvolvimento de software propõe um modelo em que especialistas de domínio e desenvolvedores trabalham em conjunto, utilizando documentos que descrevem o conteúdo do aplicativo e outras características relevantes. Assim, a aborda- 
gem documental gerencia as funções e responsabilidades dos especialistas de domínio e desenvolvedores, mas sem um envolvimento mais específico na abordagem educacional.

\subsubsection{Adaptado do modelo RUP}

No artigo de Lee, Lee, Cho, Song and Rhew [39], propõe-se um modelo empírico para processos de desenvolvimento de jogos, que pode ser facilmente aplicável ao desenvolvimento de software tradicional baseada na ISO 12207 e no RUP. Este modelo consiste em múltiplas atividades, sendo essas:

- A primeira atividade é a constituição da equipe, visto que, no desenvolvimento de jogos há integrantes de diferentes formações e atuações, tais como: designer gráfico, programadores e marketing, e assim, há muitas interações entre as diferentes funções, por isso há a necessidade da composição da equipe e suas tarefas.

- Na segunda atividade, denominada de Game Job Analysis, deve-se organizar as equipes e as funções de acordo com as plataformas e gêneros distribuídos na primeira atividade.

- Na terceira atividade, Collecting Requirements, a atividade mais importante é a competência de jogo alvo identificado pelo mercado. Assim, a equipe de planejamento e a equipe de marketing devem cooperar para coletar informações do mercado e analisá-las.

- $\mathrm{Na}$ quarta atividade, Design \& Analysis of Game Elements, a empresa vai decidir o escopo do projeto e fazer o planejamento detalhado. A primeira atividade é o planejamento da versão. Com base na atividade anterior, a equipe de planejamento e a de programação, desenvolve o design do jogo e do projeto, a fim de cooperar com a equipe gráfica/som e com a equipe de relacionamento com o cliente.

- $\mathrm{Na}$ quinta atividade, Implementation of Game Elements, a equipe de programação, e gráfico/som implementa os modelos atribuídos, bem como ferramentas de desenvolvimento interno. A parte gráfica e a de som devem ser integradas aos módulos no desenvolvimento e testadas na sincronização. Após os testes do módulo, como o desenvolvimento de software usual, a empresa deve integrar todos os resultados de implementação e a fase de testes.

- Na sexta atividade, Tests, há diferentes testes no desenvolvimento do jogo. Neste processo, a empresa deve elaborar todo o planejamento de testes e listas de verificação.

- Na sétima e última etapa, Distribution, a equipe de planejamento e de relacionamento com o cliente devem cooperar para construir o manual mestre do jogo e o manual do usuário, bem como a função on-line de ajuda.

\subsubsection{Modelo Próprio}

No trabalho de Zaibon and Shiratuddin [40], denominado "Towards developing mobile game-based learning engineering model", o objetivo principal é propor um modelo de engenharia mGBL, apresentando três etapas para o desenvolvimento de jogos móveis. Estas etapas são constituídas por: Pré-produção, Produção e PósProdução.

- Pré-Produção: constituída pelas tarefas de brainstorm, criação do conceito, criação do storyboard, criação do script do jogo, pesquisa de mercado, e planejamento de tarefas.

- Produção: etapa na qual são constituídos os elementos de multimídia, codificação do jogo, características de integração, níveis do desenvolvimento e os testes.

- Pós-Produção: constitui as tarefas de publicação, desenvolvimento, teste da versão on line, teste de dispositivos e qualidade.

\subsubsection{Considerações sobre os modelos encon- trados}

Os artigos apresentados apontam características comuns nas etapas de desenvolvimento de softwares tradicionais, como por exemplo: identificação de contexto, equipe, alocação de recursos, divisão de trabalho e testes.

Os artigos analisados estruturavam algumas ações com direcionamento para a área de jogos, como por exemplo, storyboard, área de design e etc., mesmo apresentando essas características importantes para o desenvolvimento de jogos eletrônicos.

Dessa forma, os processos encontrados foram classificados como adaptações de modelos já existentes da Engenharia de Software (ES): Cascata, Espiral e RUP. Para trabalhos que não apontavam algum processo ou modelo como base foram classificados como Próprio, assim, respondendo a primeira questão de pesquisa desta revisão.

Para a segunda questão - há algum processo de desenvolvimento que direcione a equipe, em suas atividades, à compreensão das habilidades cognitivas e psicomotoras? Não foram encontrados artigos que apresentassem ou discutissem atividades ou ações durante o desenvolvimento de jogos educacionais que contemplem as habilidades cognitivas e psicomotoras, não sendo possível responder a segunda questão de investigação.

Dessa forma, como não foi encontrada na análise dos artigos etapas que contemplassem as habilidades cognitivas e psicomotoras, definiu-se um mapeamento de características em comuns nas etapas apresentadas pelos artigos analisados, a fim de identificar atividades em comum dos artigos analisados, assim, no próximo item essas caraterísticas e suas abordagens são descritas e analisadas. 


\subsection{Mapeamento das características e abordagens dos modelos encontrados}

Detectando e analisando os modelos encontrados para o desenvolvimento de jogos eletrônicos educacionais encontrados, além de mapear os tipos de processos encontrados, algumas características comuns foram observadas, como: design, brainstorm, divisão de equipes, levantamento de requisitos, equipes multidisciplinares e testes.

Essas características foram elencadas de forma a identificar atividades relacionadas dos artigos analisados, mapeando dessa forma, quais comportamentos são comuns entre os artigos.

Para uma melhor compreensão desse mapeamento, as características foram definidas e compreendidas como:

- Design: ações para definição da parte gráfica do jogo.

- Brainstorm: discussão da ideia do jogo com os participantes da equipe.

- Equipes: divisão das ações com cada membro da equipe de desenvolvimento. jogo.

- Requisitos: discussão e definição dos requisitos do

- Equipe Multidisciplinar: equipes multidisciplinares, principalmente profissionais da área de educação.

O Quadro 3 apresenta os trabalhos que contemplam as características encontradas.

\begin{tabular}{|c|c|}
\hline CARACTERÍSTICAS & ARTIGOS \\
\hline Design & $\begin{array}{l}\text { - Lee, Lee, Cho, Song and } \\
\text { Rhew [39]. }\end{array}$ \\
\hline Brainstorm & $\begin{array}{l}\text { - Hodgson, Man e Leung } \\
\text { [38]; } \\
\text { - Lee, Lee, Cho, Song and } \\
\text { Rhew [39] e } \\
\text { - Zaibon and Shiratuddin } \\
\text { [40]. }\end{array}$ \\
\hline Equipe & $\begin{array}{ll}\text { - } & \text { Hodgson, Man e Leung } \\
\text { [38]; } & \text { Moreno-Ger, Martinez- } \\
\text { Ortiz, Sierra and Fer- } \\
\text { nández-Manjon [4] e } \\
\text { - Lee, Lee, Cho, Song and } \\
\text { Rhew [39]. }\end{array}$ \\
\hline Requisitos & $\begin{array}{l}\text { - Lee, Lee, Cho, Song and } \\
\text { Rhew [39]. }\end{array}$ \\
\hline $\begin{array}{l}\text { Equipe Multidiscipli- } \\
\text { nar }\end{array}$ & $\begin{array}{l}\text { - Hodgson, Man e Leung } \\
\text { [38]; }\end{array}$ \\
\hline
\end{tabular}

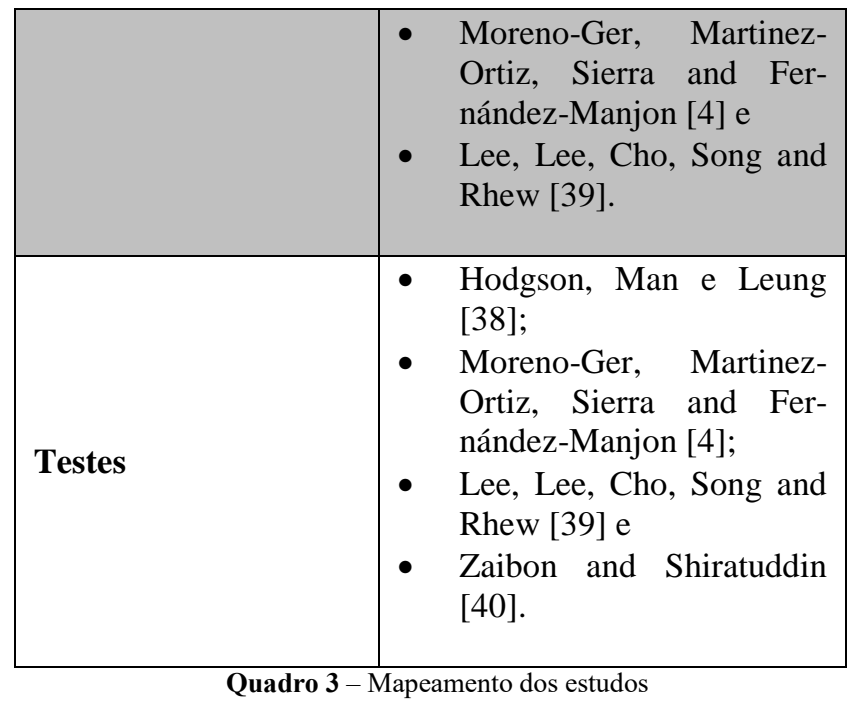

O Quadro 3 sintetizou o mapeamento das características encontradas nos artigos analisados, sintetizando propriedades e perspectivas de atividades que os modelos encontrados especificam.

Essas atividades são peculiaridades detectadas na análise de novas ações para o campo de desenvolvimento de jogos eletrônicos educacionais, visto que, decidiu-se identificar quais atividades esses artigos possuem em comum, visando gerar uma rastreabilidade desses pontos nos artigos analisados e também delinear se algum desses modelos possuem características educacionais em seus contextos, pontos importantes para a área da informática na educação, assim, tentando responder as questões desta pesquisa, principalmente a segunda.

Mesmo todos os artigos apresentando equipes multidisciplinares, nenhuma atividade ou procedimento para uma discussão referente às abordagens educacionais foi vislumbrada, principalmente as que se referem as habilidades cognitivas e psicomotoras.

Identificado essas análises sobre o mapeamento das características encontradas entre os artigos analisados, uma visão geral dos artigos, destacando, a distribuição dos artigos por anos, localidade e tipo de publicação, são apresentadas no próximo item.

\subsection{Características dos artigos}

Nessa seção, uma visão geral dos artigos, a fim de poder identificar onde estão sendo feitas as pesquisas relacionadas ao desenvolvimento de jogos eletrônicos educacionais foi mapeada.

O Gráfico 1 apresenta um panorama das localidades, onde estes artigos foram publicados, a fim de identificar aonde foram apresentados os artigos, na tentativa de reconhecer universidades, pesquisadores e centros de pesquisa da temática em questão. 
No Gráfico 1, apresenta a localidade de onde são os pesquisadores ou grupos de pesquisa encontrados nos artigos analisados.

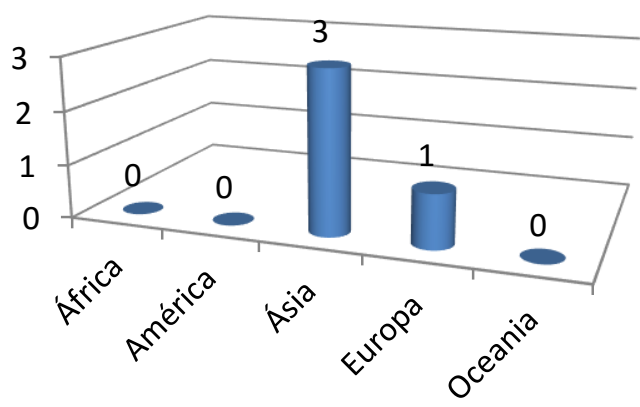

Gráfico 1 - Mapeamento de Localização das pesquisas analisadas

Pode-se observar que os pesquisadores e grupos de pesquisa, em sua maioria, 75\% pertencem à Ásia, totalizando três artigos e no que diz respeito à Europa, somente um trabalho, totalizando $25 \%$.

Mesmo sendo pesquisadores provenientes da Ásia, não encontraram-se semelhanças com as pesquisas e grupos em comum, sendo pesquisas de universidades e grupos de pesquisas diferentes.

Cabe ressaltar que os trabalhos foram publicados nos últimos nove anos (1 em 2006; 1 em 2008; 1 em 2009; 1 em 2010), deixando evidente que esta é uma área nova, em relação ao desenvolvimento de software tradicional. Um dos artigos [4] foi publicado em periódico e os outros três em conferências.

Esta subseção visou apresentar uma visão geral dos artigos, apontando características referentes à distribuição dos artigos e localização de publicação.

\section{Discussão}

Os trabalhos analisados tiveram como foco atividades que auxiliam as equipes de desenvolvimento a ter uma estruturação e um posicionamento das ações para a criação de jogos eletrônicos educacionais, porém, nenhum dos artigos analisados discute fortemente a questão educacional, principalmente, as abordagens das habilidades cognitivas e psicomotoras em seu contexto, mesmo sendo jogos eletrônicos voltados ao âmbito da educação.

Identificou-se que três dos quatro artigos apresentados, tiveram como base características de modelos da ES já existentes e uma das pesquisas apresentou um modelo próprio, mas, com ações subliminares de atividades presentes nos modelos existentes.

Nenhuma pesquisa propôs ações que proporcionam uma discussão sobre a maneira pela qual as equipes poderiam identificar as ações educacionais (habilidades cognitivas) em seu desenvolvimento.
Sendo assim, algumas indagações são despertadas, será que as habilidades cognitivas e psicomotoras não são importantes em um jogo eletrônico educacional? Os pesquisadores [33] defendem essa importância argumentando que se um jogo eletrônico voltado ao processo de ensino e aprendizagem contemplasse essas habilidades, o processo cognitivo do aprendiz seria estimulado e assim, a aprendizagem ocorreria de forma natural. Consequentemente, as habilidades cognitivas e psicomotoras são de grande importância para a área.

Dessa forma, se a equipe de desenvolvimento conseguisse identificar essas habilidades, poderiam exteriorizálas nas ações dos jogos, no desenvolvimento de cenários, dos personagens, e de procedimentos didáticos, apontando assim, a importância da área pesquisada, principalmente para a informática na educação.

Mesmo com os artigos analisados envolvendo equipes multidisciplinares, percebeu-se, que essas equipes dão um suporte educacional conteudista, não havendo uma relação de discussão nas equipes para o desenvolvimento das habilidades que um jogo eletrônico educacional precisa ter.

Assim, os resultados dos artigos analisados posicionam e ajudam as equipes de desenvolvimento a terem uma estruturação de atividades, proporcionando: a discussão de ideias, divisão de tarefas entre os integrantes das equipes, levantamento de requisitos e testes no decorrer do desenvolvimento do jogo em si. Porém, ações educacionais não são discutidas em etapa alguma do processo de desenvolvimento.

Desta maneira, faz-se necessário a discussão de novas ações que possibilitem a contextualização das habilidades cognitivas e educacionais nas etapas do desenvolvimento do jogo eletrônico educacional, contextualizações estas, importantes para a área da informática na educação, pois podem auxiliar no desenvolvimento cognitivo do aprendiz com base em um contexto tecnológico.

A integração da estruturação existente com essa contextualização educacional pode auxiliar os profissionais de área a assimilar essas contextualizações em visões técnico-pedagógicas.

Com esta revisão sistemática de literatura, detectou-se a falta de ações educacionais que auxiliem equipes de desenvolvimento de software a contextualizá-las no desenvolvimento de jogos eletrônicos educacionais, auxiliando os profissionais da área de exatas a compreender essas adversidades à luz das possibilidades educacionais. Se os desenvolvedores entenderem um pouco mais as práticas pedagógicas, ou até mesmo forem auxiliados na identificação e compreensão de algumas habilidades cognitivas e psicomotoras, essas ações poderiam ser inseridas no jogo eletrônico educacional, e assim, auxiliar no desenvolvimento de habilidades necessárias nos aprendizes. 


\section{Conclusão, Limitações e Trabalhos Futuros}

O objetivo da revisão sistemática foi responder as seguintes questões: 1) Quais processos de desenvolvimento de software estão sendo utilizados para a construção de jogos eletrônicos educacionais?; 2) Há algum processo de desenvolvimento que direcione a equipe, em suas atividades, à compreensão das habilidades cognitivas e psicomotoras?

Respondendo a primeira questão, foram encontrados os modelos de processos de softwares tradicionais adaptados para o desenvolvimento de jogos eletrônicos educacionais, sendo: cascata, espiral e RUP e somente um modelo caracterizado como próprio.

$\mathrm{O}$ aporte teórico e o motivacional apresentados demonstram que os modelos encontrados não são suficientes para o contexto atual, visto a diversidade de ações e proposições educacionais que um jogo eletrônico educacional deve conter.

Além disso, apesar de alguns modelos incluírem equipes multidisciplinares, não percebeu-se em nenhum o propósito de definir e estruturar ações voltadas ao processo de ensino e aprendizagem e, os profissionais envolvidos nas equipes de desenvolvimento, em sua maioria, não discutem ou abordam as proposições cognitivas ou motoras nas etapas de desenvolvimento.

Os modelos adaptados propostos visaram auxiliar as equipes de desenvolvimento a ter uma estruturação e um posicionamento das ações para a criação de jogos eletrônicos educacionais, contudo, nenhum desses artigos discute de forma esquematizada a questão cognitiva e educacional em seu contexto, para que as equipes discutem e entendam essas questões, mesmo sendo jogos eletrônicos voltados ao âmbito da educação.

A identificação da falta de etapas ou atividades no processo de desenvolvimento destinado à compreensão das habilidades cognitivas e psicomotoras em um processo de desenvolvimento de um jogo eletrônico educacional é preocupante, uma vez que, essas abordagens são de grande relevância para o desenvolvimento de ações pedagógicas.

Dessa forma, não foi possível responder a segunda questão de pesquisa, visto que não foi encontrado um processo de desenvolvimento para jogos eletrônicos educacionais que possibilite a compreensão das habilidades cognitivas e psicomotoras.

Assim, evidencia-se a importância de se propor um processo de desenvolvimento de jogos eletrônicos educacionais que contemple questões cognitivas e que possibilite à equipe de desenvolvimento identificar as habilidades cognitivas e psicomotoras, auxiliando desse modo as equipes desenvolvedoras na identificação de ações educacionais e cognitivas, bem como, proporcionar a cons- trução de produtos finais (jogos) que contribuam para o processo de aprendizagem do público pretendido.

No entanto, apesar da relevância do tema há um número reduzido de artigos que o abordam. Dos 956 trabalhos resultantes de uma pesquisa em 7 bases de dados científicas relevantes, apenas 4 artigos científicos estavam diretamente relacionados com o contexto pesquisado. A maioria dos artigos tratava de avaliações de jogos eletrônicos educacionais existentes, em um contexto para o processo de ensino e de aprendizagem. A justificativa para esse número reduzido pode estar no fato de que as pessoas podem usar termos diferentes para processos de desenvolvimento e jogos educacionais ou pela área de jogos eletrônicos ser relativamente nova em relação a outras abordagens de desenvolvimento de softwares diversos.

Em relação a validação dos dados encontrados, apenas um pesquisador participou como revisor para o processo de seleção e extração dos dados, consequentemente, não houve tratamento de ameaças a validade de decisões subjetivas em relação às análises dos artigos encontrados.

Como resultado desta pesquisa, foi desenvolvido um Processo de Desenvolvimento de Jogos Eletrônicos Educacionais, que contempla o entendimento das ações educacionais e das habilidades cognitivas e psicomotoras, a fim de auxiliar as equipes de desenvolvimento na contextualização dessas ações durante o desenvolvimento de um jogo eletrônico educacional e um ambiente computacional de auxilio as equipes de desenvolvimento à identificarem e padronizarem ações para o desenvolvimento de um jogo eletrônico educacional, visando à compreensão das habilidades cognitivas e psicomotoras em seu desenvolvimento.

\section{Agradecimentos}

À Fundação Araucária de Apoio ao Desenvolvimento Científico e Tecnológico do Paraná, agência de fomento brasileiro, pelo apoio financeiro.

\section{Referências}

[1] T. M. Connoly, E. A. Boyle, E. MacArthur et al.. A systematic literature review of empirical evidence on computer games and serious games. Computers \& Education, 59, 661-686, 2012.

[2] G. D. Correa, G. A. Assis, M. Nascimento et al. GenVirtual: um Jogo Musical para Reabilitação de Indivíduos com Necessidades Especiais. Revista Brasileira de Informática na Educação Volume 16 - Número 1 - Janeiro a Abril de 2008, p.9-17, 2008. 
[3] V. Vangsnes, N.T.G Økland, R. Krumsvik. Computers games in pre-school settings: Didactical challenges when commercial educational computer games are implemented in kindergartens. Computers \& Education, 58, 1138-1148, 2012.

[4] P. Moreno-Ger, I. Martinez-Ortiz, J. L. Sierra, B. Fernández-Manjón. A Content-Centric Development Process Model. Computer, 41(3), 24-30, 2008.

[5] T. Hainey, T. Connolly, M. Stansfield, E. Boyle. The differences in motivations of on line game and offline game players: A combined analysis of three studies at higher education level. Computers \& Education, 57, 2197-2211, 2011.

[6] P. Lavín-Mera, P. Moreno-Ger, B. FernándezManjón. Development of Educational Videogames in m-Learning Contexts. In: Second IEEE International Conference on Digital Game and Intelligent Toy Enhanced Learning, pp. 44-51, 2008.

[7] A .S. Perucia, A. C. Berthêm, G. L. Bertschinger et al. Desenvolvimento de Jogos Eletrônicos: Teoria e Prática. São Paulo: Novatec, 2007.

[8] M. Rodriguez-Cerezo, J-L Sierra Gomez-Albarr’n. From Collections of Exercises to Educational Games: A Process Model and a Case Study. In 2011 IEEE 11th International Conference on Advanced Learning Technologies, 282-284, 2011

[9] S. Gomes, E. .G. Wanderley. Elicitando requisitos em projetos de Software Educativo. In $I X$ Workshop de Informática na Escola- WEI. 119130, 2008.

[10] T. Mandel The Elements of user interface. John Wiley and Sons: New York, 1997.

[11] M. H. Pietruchinski, J. Coelho Neto, A. Malucelli, S. Reinehr. Os jogos educativos no contexto do SBIE: uma revisão sistemática de Literatura. In: XXII Simpósio Brasileiro de Informática na Educação e XVII Workshop de Informática na Escola, Aracaju-SE, Brasil, 2011, pp. 476-485.

[12] C. Silva, A. Calisto, D. Barbosa. Proposta de um processo de desenvolvimento de jogos educativos. In: Anais do XXI Simpósio Brasileiro de Informática na Educação, João Pessoa, PB, Brasil, 2010.

[13] D. S. R Vosgerau. A Tecnologia Educacional face à evolução das correntes educacionais: as contribuições da psicologia cognitiva. Revista Contrapontos. V.7, N.2, p. 269-281, mai-ago, 2007.

[14] A. Littlejohn, I. Falconer, L. Mcgill. Characterizing effective eLearning resources. Computers \& Education, 50 (2008), pp. 757-771.
[15] W. S. Humphrey. Managing the Software Process. MA: Addison-Wesley Publishing Company Inc., 1989.

[16] I. Sommerville. Engenharia de Software. São Paulo: Pearson Addison-Wesley, 2007.

[17] P. Kruchten. The Rational Unified Process: an Introduction. Addison Wesley, $2^{\text {nd }}$ ed. 2000.

[18] M. Levy, O. Hazzan. Knowledge management in practice: The case of agile software development. Workshop on Cooperative and Human Aspects on Software Engineering, 2009. CHASE '09. ICSE, vol., no., May, 2009. pp.60-65.

[19] A. Souza, I. Kafure. O fator emocional no desenvolvimento de jogos. In: SBC Proceedings of XI SBGames - Art \& Design Track - FullPapers Brasília - DF, p. 130-133, 2012.

[20] A. Santos, V. A. Góes, L. F. Almeida. Metodologia OriGame: um processo de desenvolvimento de jogos. In: SBC Proceedings of XI SBGames - Art \& Design Track - FullPapers - Brasília - DF, p. 125-132, 2012.

[21] P.C. Ilha, D. Cruz. Jogos Eletrônicos na educação: uma pesquisa aplicada do uso de SimCity 4 no Ensino Médio. In Anais XXVI Congresso da SBC. Campo Grande - MS, 2006.

[22] R. J. Sternberg. Psicologia cognitiva. Trad. Anna Maria DalleLuche, Roberto Galman. Rev. Téc. José Mauro Nunes - São Paulo: Cengage Learning, 2010.

[23] R. O. Nascimento. Processos Cognitivos como elementos fundamentais para a educação crítica. Ciências \& Cognição. v.14 (1): p.265-282, 2009.

[24] R.J. Fernandes; G. Santos Junior. The SIMS: Jogo Computacional como Ferramenta Pedagógica na construção do Conhecimento Matemático. Revista Eletrônica TECCEN, Vassouras, v. 5, n. 1, jan./abr., 2012 , pp. 21-36.

[25] J. E. Garrido, V. M. R. Perrichet, M. D. Lozano, et al. Mobility and Memory Trainning through Movement Interaction. In: Proceedings of Federated Conference on Computer Science and Information System, pp. 883-889, 2012.

[26] A. Meur, L. Staes. Psicomotricidade: educação e reeducação. Trad. Ana Maria Izique Galuban e Setsuko Ono. São Paulo: Manole, 1991.

[27] H. Walon. Évolution Psychologique de l'enfante. Colin, Paris, 1968.

[28] S. Carrijo, H. M. Tavares. A contribuição de psicomotricidade no trabalho psicopedagógico. Re- 
vista da Católica, Uberlândia, v.3, n.6, p. 385-397, 2011.

[29] E. Assunção José, M. T. Coelho. Problemas de Aprendizagem. 8. Ed. São Paulo, SP: Ed. Ática, 1996.

[30] M. R. Tarouco, L. C. Roland, M. C. Fabre et al. Jogos Educacionais. Revista Novas Tecnologias na Educação, v.2, no. 1, março, p. 1-7, 2004.

[31] C. Santiago, I. A. Batista, R. R. Padovani et al. A proposal of Cognitive Classification of Eletronic Games. In: SBC Proceedings of XI SBGames - Art \& Design Track - FullPapers - Brasília - DF, p. 85-92, 2012.

[32] M. Pinto, S. C. Botelho, R. C. Souza, et al.. Plataforma Saberlândia: Integrando Robótica e Multimídia no Desenvolvimento de Jogos Educacionais. In: Proceeding of Simpósio Brasileiro de Jogos e Entretenimento (SBGAMES), ComputingTrack FullPapers, Belo Horizonte, MG, 2008.

[33] R. Rieder, E. M. Zanelatto, J. D. Brancher. Observação e Análise da Aplicação de jogos educacionais bidimensionais em um ambiente aberto. In IX Taller International de Software Educativo (TISE), Santiago - Chile, 61-66, 2004.

[34] J. Novak. Desenvolvimento de games. Trad. Pedro Cesar de Conti; Ver. Tec. Paulo Marcos Figueiredo de Andrade - São Paulo: Cengage Learning, 2010 .

[35] K. Corti Games-based Learning: a serious business application. Pixe Learning, 2005.

[36] B. Kitchenham. Procedures for Performing Systematic Reviews. Keele UK Keele UniversityPublisher: Citesser, 33(TR/SE-0401), 28, 2004.

[37] C. Wohlin, P. Runeson, M. Höst, M.C. Ohisson, B. Regnell, A. Wesslén. Experimentation in Software Engineering. Springer, 2012, XXIV, 236p.

[38] P. Hodgson, D. Man, J. Leung. Managing the Development of Digital Educational Games. 2010 Third IEEE International Conference on Digital Game and Intelligent Toy Enhanced Learning, 191-193, 2010.

[39] S. H. Lee, G. H.; Lee, H. H. Cho et al. An Emprirical Model of the Game Software Development Processes. In Proceedings of the Fourth International Conference on Software Engineering Research, Management and Applications (SERA), 371- 377, 2006.

[40] B. Zaibon, N. Shiratuddin. Towards development mobile game-based learning engineering model.
In World Congress on Computer Science and Information Engineering, 649-653, 2009. 